\title{
UNA IMPORTANTE CONTRIBUCIÓN DE ROBERT RICARD AL ESTUDIO DE SOR JUANA INÉS DE LA CRUZ
}

\author{
P O R
}

\section{JOSE ROJAS GARCIDUEÑAS}

$\mathrm{A}^{\mathrm{N}} 1954$ Robert Ricard dictó, en la Sorbonne, tres lecciones sobre circunstancias particulares, que adelante se verán, parece pertinente recordar aquel breve pero importante cursillo, que publicó en un folleto de 45 páginas el Instituto de Altos Estudios de América Latina, en París. ${ }^{1}$

Presentar a Robert Ricard, hoy Director del Institut Hispanique, al público de estos Anales sería de tal manera superfluo que no hay para qué considerarlo, pues los lectores de estos Anales son estudiosos del arte y de las letras de México y Ricard ha sido uno de los investigadores cuyos escritos ya se consideran básicos en la bibliografía histórica de nuestro país. Lo que sl cabe decir, porque siempre está bien lo que es justo y debido, es poner de manifiesto nuestro agradecimiento al doctor Ricard por su profundo interés y sus luminosas enseñanzas en muchos aspectos de la historia de México, a partir de aquella magnífica contribución en ese campo, escrita hace ya treinta años y que sigue siendo un firme apoyo en el conocimiento de nuestro siglo xvi. ${ }^{2}$

1 Robert Ricard: Une poêtesse mexicaine du XVIIe siecle, Sor Juana Inés de la Cruz. Université de Paris. Institut des Hautes Etudes de l'Amérique Latine, Paris, s.d. [E.l curso está fechado, al final: Juillet 1954].

2 Me refiero a: La conquéte spirituelle du Mexique, cuya edición francesa apareció en 1933, y su traducción, en México, en 1947. 
El estudio sorjuanístico al que, principalmente, me voy a referir aquí, está dividido en tres lecciones.

La primera de ellas: Juana de Asbaje et Sor Juana Inés de la Cruz (1648-1691), como su título indica, es un resumen biográfico de la poetisa. El autor definitivamente rechaza, como fecha del nacimiento de Juana, la tradicional de 1651 y acepta la de 1648, refiriéndose a lo ya examinado por Alfonso Méndez Plancarte (Obras completas de Sor Juana Inés de la Cruz, t. x, México, 1951. p. LII-LiII, nota 1), con muy justas observaciones, como aquellas de que, en rigor, el acta de bautizo descubierta en los archivos parroquiales de Chimalhuacán se refiere únicamente a cierta Inés, sin filiación, bautizada el 2 de diciembre de 1648, pero que el hecho de que los nombres de los padrinos sean los del hermano y de la hermana de Isabel Ramírez, madre de la futura Sor Juana, autoriza la opinión de que tal acta sea la del bautizo de la célebre monja. Por otra parte -continúa Ricard-, el P. Calleja habla del viernes 12 de noviembre de 1651, como fecha del nacimiento de Sor Juana, siendo así que aquel año el 12 de noviembre cayó en domingo, lo que debe ponernos en guardia contra la exactitud de la información; todo lo cual concurre a robustecer la suposición de que Sor Juana nació el 12 de noviembre de 1648. 3

Los datos relativos a la infancia son los que la misma monja dio a conocer en su carta a Sor Filotea; en cuanto a su estancia en Palacio, como dama de la virreina, dice pintorescamente Ricard: "Née vers la fin de 1648, Juana de Asbaje a environ quinze ans. Elle fait la conquête de la Marquise et elle devient la «coqueluche» du palais"; * luego su breve estancia con las monjas de Santa Teresa, su regreso a Palacio y, finalmente, su ingreso al convento de San Jerónimo el 24 de febrero de 1669, a lo cual llama Ricard la "primera conversión" de Juana.

Esos términos de primera y segunda conversión Ricard los refiere, con las necesarias distinciones, a una evolución espiritual como la ocurrida en Pascal: "Nous assistons dans l'un et l'autre cas à l'histoire de deux esprits d'une extraordinaire précocité qui renoncent peu à peu à tout ce qui pouvaient leur apporter le prestige et le rayonnement d'une intelligence privilégiée, pour s'élever de ce que Pascal nomme précisément «l'ordre des esprits à ce qu'il nomme encore cl'ordre de la charité». Pour Pascal, on le sait, cette ascension ne se fit pas d'un seul coup.

3 Une poétesse mexicaine... p. 12, nota 1.

\& Op. cit., p. 3 . 
Il en fut de même pour Sor Juana. Elle aura, elle aussi, comme Pascal, une seconde conversion. Mais la première constitue une étape décisive -ne serait-ce que parce qu'elle prépare naturellement la seconde-, et elle ne manque pas de susciter bien des interrogations". 5

El problema intriga profundamente a Ricard, después de brevísimo resumen de la vida de Sor Juana en el convento, y acaba por atribuir la "segunda conversión" a la crisis desencadenada por la Carta Atenagórica de Sor Juana y sus consecuencias: la Carta del Obispo Santa Cruz a la monja y la Respuesta que ella dio. El problema es demasiado complejo para quedar, no digamos elucidado, pero ni siquiera tratado, en tan breve espacio como el que Ricard pudo dedicarle en el plan de su cursillo, pero sólo el hecho de haberlo planteado indica cuánto ha pensado y estudiado la vida de Sor Juana. Ricard da, pues, una gran importancia en la segunda crisis de Sor Juana (para decirlo al modo barroco de su tiempo), a la crítica de la que ella hizo al Padre Vieira; problema que el mismo hispanista ya había expuesto en otro estudio particular ("Antonio Vieira et Sor Juana Inés de la Cruz", en Bulletin des. études portugaises, t. xII, 1948), dejando en segundo término, y refutando que hubiese sido decisiva en aquella dicha crisis, la influencia del jesuita Núñez de Miranda, confesor de Sor Juana, como lo sintetiza al final de esta parte de su estudio que comento, diciendo: "On a beaucoup discuté sur le rôle joué dans la seconde conversion de Sor Juana par son confeseur, le Père Jésuite Antonio Núñez de Miranda. Quelle qu'ait été l'influence du $\mathrm{P}$. Núñez sur sa dirigée, il reste que la seconde conversion résulte d'une volonté personnelle de Sor Juana. Sur le P. Núñez de Miranda, cf. Joseph de Guibert, S. J., La Spiritualité de la Compagnie de Jésus, ouvrage posthume, Rome, 1953, p. 313 et p. 375, n. 31."

Sin duda el problema es difícil y muy discutible, pero el planteamiento de Ricard es serio y cuidadoso, por lo mismo digno de elogio tal examen y la forma en que lo hace. Qué diferencia con las afirmaciones desmesuradas y con tanta frecuencia imaginativas de Ezequiel A. Chávez, que hizo derroche de citas de psicólogos y autoridades para convertir a la monja poetisa en un caso de ascensión mística ininterrumpida y afirmar que sus años finales fueron un puro arrebato, tan deshumanizado o sobrenatural, que la monja estuvo a punto de perder "la

\footnotetext{
5 op. cit., p. 3 y 4.

6 op. cit., p. 13, n. 5 .
} 
personalidad misma, víctima de la obediencia total por la que, abdicando de sí propia, el alma se suicida". ${ }^{7} \mathrm{Y}$ también afirma que ya en ese plano, o en ese vértigo, el concepto de vida superior en la monja y el concepto análogo del Padre Núñez de Miranda "subían al mismo ápice y tenían que llevar arriba el alma, a la Luz Infinita, a la Sabiduría Infinita, a la Poesía Inefable, al Amor Excelso, a la Vida Increada y Perfecta ..." 8 Con justicia, aunque con mucha moderación y cortesía, comentando ese libro don Genaro Fernández Mac Gregor denunciaba tales extravíos diciendo que "Para formar el señor Chávez su juicio comparativo sobre los valores psíquicos del P. Antonio y de Sor Juana, emplea criterios de mística india, no de mística cristiana, que son los que por el credo de los dos personajes, y por la índole de los tiempos en que vivieron, se les debiera aplicar..." Fernández Mac Gregor niega el misticismo de Sor Juana, pues no encuentra que su obra "revele otra cosa que una piedad cristiana, natural en una religiosa; una devoción que no se eleva, ni mucho menos, al éxtasis ... Su vida -continúa diciendo-, por otra parte, confirma esta manera de ver, pues se desarrolla en redor del drama interior actuado entre sus dos magnas tendencias: la frivolidad y la reflexión." $10 \mathrm{Y}$ señala, indirectamente, que la actitud de Ezequiel Chávez es, en cierto modo, el extremismo del converso, recordando el cambio entre el positivista que era cuando se redactaba y aparecía México y su Evolución Social (es decir, hacia 1910, cuando Chávez era Subsecretario de Instrucción Pública), en contraste con el espiritualista, ferviente católico y casi místico que escribió el citado libro: Ensayo de psicologia de Sor Juana Inés de la Cruz . . , hacia los años de 1925-30.

Mucho llevo de andar fuera de la vía que debiera, en este artículo cuyo objeto es tratar del estudio sorjuanístico de Robert Ricard, pero la verdad es que cuesta trabajo detener la pluma, cuando fluyen los comentarios y atraen las diversas perspectivas en el examen de esos problemas, que cautivan como cautiva todo lo que se relaciona con aquella excepcional mujer. En cuanto al papel que cerca de ella tuvieron Núñez de Miranda, Santa Cruz y otros personajes, espero que algo más sabre-

7 Ezequiel A. Chávez: Ensayo de psicología de Sor Juana Inés de la Cruz y de estimacion del sentido de su obra y de su vida para la historia de la cultura y de la formación de México. Casa Editorial Araluce, Barcelona, 1931, p. 439.

8 Op. cit., p. 402.

- Genaro Fernández Mac Gregor: La Santificación de Sor Juana Inés de la Cruz. Editorial Cultura, México, 1932. p. 65.

10 Op. cit., p. 42. 
mos cuando nuestro colega de este Instituto, Francisco de la Maza, prosiga y concluya su estudio sobre las personas que rodearon a Sor Juana y de un modo $u$ otro intervinieron en su vida. Quede aquí este punto de digresión y volvamos al tema.

La segunda lección de Ricard se titula: La poésie savante: Le "Sueño". Esta parte es, sin duda, la más original del curso y por eso, antes que hacer comentarios, parece conveniente hacer citas de sus párrafos fundamentales, no importando que los fragmentos citados sean más largos que lo que en tales casos se acostumbra pues, al fin y al cabo, el lector saldrá ganando con tener más proporción del buen estudio de Ricard que del resumen deficiente que yo podría hacer de él.

Comienza por recordar que la poesía de Sor Juana se da en dos aspectos: "l'aspect savant, culto et cultista, et l'aspect populaire ou semi-populaire" en lo cual, añade, Sor Juana no escapa a una tendencia fundamental de la literatura española, que no conoce frontera entre lo profano y lo sagrado, y asimismo, en los mayores clásicos, a veces en la misma obra se encuentra la tradición culta más sutil y refinada con la robusta y sabrosa tradición popular. Ricard separa los dos géneros o modalidades de la poesía de Sor Juana: en esta segunda lección el aspecto culto, dejando para la tercera el aspecto de poesía popular.

Como ejemplo más característico de la poesía culta sorjuanista considera el Primer Sueño, y para su análisis distingue en ese poema tres partes, que serían estas: I. Le sommeil du "cosmos". II. L'homme, le rêve et le "cosmos": a) description physiologique et psychologique du sommeil et du rêve; b) récit du rêve. III. Le réveil de l'homme et le réveil $d u$ "cosmos". He preferido transcribir y no traducir esos enunciados porque me parece que ellos mismos, en francés, dan mejor idea del pensamiento de Ricard, y con más claridad, cuando llama sommeil a la primera parte y dice rêve para referirse al contenido de la segunda. Por igual motivo también conservo en el idioma original los párrafos que citaré más adelante.

En cuanto al sueño del "cosmos", Ricard resume el contenido de los primeros 150 versos del poema; él no señala exactamente la división entre una parte y otra (división que, desde luego, en el poema no existe), pero es evidente, y creo con toda razón, que él piensa que los versos 140-149 son el final de la primera parte, y yo diría que son más bien una transición, un "puente", como se suele llamar en música a los compases que separan y ligan, al mismo tiempo, al primero con el segundo tema de una sonata. 
La segunda parte es l'homme, le rêve et le "cosmos"; es decir, según Ricard hay un cambio de esencia o de naturaleza en el objeto del poema, que pasa del sommeil al rêve, pero, además, distingue claramente la descripción fisiológica y psicológica "du sommeil et du rêve" y, aparte, lo que denomina "récit du rêve". Parece, según tal división, que la dicha descripción empezaria en el verso 151: "El conticinio casi ya pasando..." y sigue hasta el verso 296: "que con similitud en sí gozaba"; aquí empezaría le récit du rêve, en el verso 297, cuando el alma: “... juzgándose casi dividida" se eleva sobre todas las cimas artificiales y naturales y trata de ver todo para entender todo. Eso no es posible por una percepción directa, pues la infinita multiplicidad de imágenes produce confusión; el espíritu, entonces, procede a un análisis y a un método, tratando de llegar al conocimiento por especies o categorías; sin embargo, el método no basta para conocer la esencia de los fenómenos; el anhelo de conocimiento es audaz pero las audacias desmedidas son peligrosas, como lo enseña el caso de f́caro, por más que esa misma celebridad es nociva a pesar de que igualmente se conoce el castigo. De modo que esa parte llegaría, asi, hasta el verso 826: "su ejecución, que no a lo escarmentado". Del verso siguiente: "Mas mientras entre escollos zozobraba", hasta el verso 852: "las cadenas del sueño desataban", yo considero que hay otro "puente", y del verso 853 en adelante es lo que Ricard considera como tercera parte, el despertar del hombre y del cosmos, hasta que ambos confluyen en el último verso, 975, "el Mundo iluminado, y yo despierta", con el que termina el poema. Creo haber seguido fielmente la idea de Ricard y únicamente he tratado de precisar las zonas límites de cada una de las partes del Primer Sueño.

Esas ideas sobre las partes del poema significan conceptos de la organización del mismo, de alli la importancia de conocerlas con precisión. La concepción de Ricard, a tal respecto, es, como se ha podido ver, de una gran sencillez $y$, al mismo tiempo, ayuda mucho a percibir la estructura completa, en su todo y en sus partes, del poema. No es aquí lugar para hacer sobre esto las consideraciones extensas que serían menester a propósito de esa magnífica y muy elaborada arquitectura (si fue producto de larga reflexión o de rápida genial intuición es otra cosa que, además, en este caso no es averiguable), quede ello para el momento de analizar esa obra, pero sí debe hacerse notar que a tal análisis mucho ayudarán las claras y justas observaciones de Ricard. El mismo señala, en un párrafo, algunas objeciones a lo que otros estudiosos habian dicho o propuesto como lineamientos de construcción del poema de Sor 
Juana: Alfonso Méndez Plancarte distingue doce partes, lo cual, sin duda, cra fragmentar demasiado; Ezequiel Chávez encontraba seis, pero lo grave es que él creía distinguir en el poema seis "sueños", lo que Ricard censura muy justamente: "on peut lui reprocher d'avoir introduit dans sa division une symétrie exagérément artificielle: Sueño de la noche, Sueño del Sueño universal, Sueño del Sueño del hombre, Sueño de los Sueños, Sueño del conocimiento, Sueño del despertar. Titres d'ailleur amphibologiques, par suite du double sens du mot sueño en espagnol..." 11 Y Ricard alude a la interpretación de Karl Vossler y a la división de Pfandl, explicando así, o mejor aludiendo a la sencillez de su propia idea de la composición: "Karl Vossler a donné une interprétation plus brève que celle de $M$. Méndez Plancarte, et parfois erronée, mais où il a renoncé à dégager des divisions fermes. C'est peut-être un autre excès, car ce déroulement ininterrompu n'empêche pas l'oeuvre d'être véritablement composée. Aussi ai-je préféré me rapprocher de Ludwig Pfandl, qui se borne à cinq divisions: le mystère du rêve, le mécanisme du rêve, la matière du rêve, le réveil de l'homme, le réveil du monde. Encore ai-je simplifié cette division de la manière que nous avons vue, en me limitant à trois grandes parties dont j'ai seulement divisé en deux la partie centrale." $Y$ añade en nota: "Finalement, le plan que je crois discerner est peu différent de celui que propose M. Emilio Carilla dans un article de la Revista de filología española, XXXVI, 1952, p. 295." 12

Es el Sueño, de Sor Juana, uno de los más claros y eminentes ejemplos de poesía culta, no en el sentido usual de influencia gongorina (lo "culto" satirizado por Quevedo: "si culto quieres ser en sólo un día", la "culta latiniparla", etc.), sino en el de poesía que supone y se basa en cierto nivel, o más bien en alto nivel de cultura y de conocimientos. En este aspecto, en los conocimientos que el mismo poema revela, uno de los puntos que han dado lugar a discusión es el de la influencia cartesiana, que algunos críticos creen percibir claramente, por entender la mención de un "método" como referencia directa al sistema de Descartes y que, en cambio, otros niegan con no pocas razones. Ricard se muestra en esto muy cauteloso, en verdad él no cree que el cartesianismo haya penetrado en México antes del siglo xvir.

En cuanto a la forma literaria, Ricard alude a las evidentes influencias de Góngora y otros poetas de la época.

11 Ricard: Une poettesse..., p. 20.

12 Op. cit., p. 20 y p. 28, n. 5 . 
Más importa su observación de que el poema tiene una tonalidad más filosófica que propiamente religiosa, y añade que "hasta se puede decir que es apenas cristiano: al lado de numerosas menciones sacadas de la tradición grecolatina, el Evangelio sólo aparece una vez, y eso indirectamente cuando evoca a San Juan, el Aguila Evangélica (verso 681: "la que Aguila Evangélica, sagrada ..."). Dios mismo no es nombrado y si aparece varias veces es mediante alusiones y designaciones estrictamente filosóficas: alto Ser, Causa Primera, Sabia Poderosa Mano, Eterno Autor. Apenas se va más allá de un vago espiritualismo deísta, carácter que puede explicarse por el género al que pertenece el Sueño. $Y$ aquí es donde se plantea la cuestión de su originalidad.". ${ }^{13}$

Las dos páginas que siguen son especialmente importantes puesto que contienen las ideas de Ricard que él opone a las de Ludwig Pfandl; tratándose de tales autoridades y de tan importante cuestión, juzgo preferible transcribir literalmente los párrafos respectivos:

"De l'influence manifeste, presque écrasante, de Góngora, faut-il d'abord conclure que le Sueño n'est pas original? Cette influence se borne au vêtement extérieur; conception et sujet lui échappent. Góngora ne semble pas avoir été particulièrement attiré par les problèmes psychologiques et cosmologiques. Sor Juana a voulu décrire en vers gongorinos une expérience personnelle, et elle l'a fait avec une étonnante capacité d'analyse et d'introspection, d'autant plus remarquable que ses théories ne lui facilitaient pas beaucoup cette analyse. Mais il faut nous garder contre deux tentations, d'inégale gravité. La première est facilement explicable, puisqu'il s'agit d'une vision que l'auteur présente comme un songe réel: elle consiste à y chercher une révélation de l'inconscient de Sor Juana, un défoulement, et à l'interpréter en termes de libido conformément à des principes freudiens plus ou moins bien compris. C'est la tentation à laquelle a succombé Ludwig Pfandl. Son recours à la psychanalyse semble en effet beaucoup moins légitime que dans son livre sur Jeanne la Folle, car celle-ci relevait bien plus certainement de la méthode qu'il a appliquée ensuite à la poétesse mexicaine. Quoi qu'on pense de la psychologie analytique en elle-même, il y a beaucoup d'imprudence, sauf dans le cas de personnages indiscutablement fous, à y recourir de manière rétrospective, pour expli-

13 op cit., p. 22. 
quer un texte forcément figé et dû à un auteur disparu depuis deux siècles et demi. La seconde tentation, qui est connexe d̀ la première, c'est de croire que Sor Juana a voulu nous conter un rêve réel, en d'autres termes de faire de cette oeuvre d'art un témoignage... Quelles qu'aient été la puissance d'analyse et la mémoire de Sor Juana, il ne semble pas possible qu'un texte aussi long et aussi compliqué, et écrit en vers, ne soit rien d'autre que le simple récit d'un songe réel. Elle dit bien qu'elle faisait des vers en dormant, mais il ne faut pas oublier que le Sueño en compte près de 1000 . Pour moi, il y a eu toute une élaboration nouvelle, fruit d'une méditation approfondie, opérée elle-même sur des souvenirs d'une exceptionnelle précision, et on doit envisager une véritable recréation littéraire, où il est impossible de faire le départ entre ce qui provient des rêves mêmes de Sor Juana et les additions et modifications qu'elle a pu introduire en écrivant son poème.

"Ajoutons entre parenthèses que cette interprétation rend encore moins acceptable l'exégèse psychanalytique, mais ce n'est aucunement pour écarter celle-ci que je la propose, c'est simplement parce qu'elle est celle qui me paraît la plus conforme à la vraisemblance psychologique et littéraire. Il n'en reste pas moins que le rêve et en particulier un état intermédiaire entre le rêve et la méditation, plus intellectuel que ce qu'on appelle la crêverie", ont joué un grand rôle dans la vie psychologique de Sor Juana. Elle nous explique, dans sa réponse à Sor Filotea, comment son esprit, toujours en éveil, travaillait et réfléchissait sur les moindres choses, et il n'est pas étonnant que ce travail de réflexion se soit prolongé pendant le sommeil ..." 14

Personalmente yo creo que, si alguna base real tiene el Primer Sueño, es decir si alguna parte de él es un recuerdo de un sueño que Sor Juana tuvo, ello sería apenas algo más que un punto de apoyo, un extremo de trampolín del que se lanza de lleno a la creación puramente poética $y$ profundamente intelectual; de su naturaleza propiamente onfrica yo no veo que algo haya quedado y nada quedó de su proceso característico que es, fundamentalmente, ilógico, ya que el soñar es un proceso subconsciente ajeno a construcciones lógicas que son procesos conscientes. Para decirlo con mejores palabras, las de un agudo psicólogo y gran literato: “... Le rêve est presque toujours absurde, et d'une absurdité 14 Op. cit., pp. 22-24. 
spéciale, incohérente et déroulé selon ses associations toutes passives dont la marche diffère même de celles des ordinaires associations passives, conscientes ou subconscientes..."15 Por lo demás, el propio Ricard finalmente relaciona el Sueño de Sor Juana con otros ejemplos del mismo género literario, como adelante diré, pero antes es preciso tratar, o siquiera mencionar, algunas de las ideas de Pfandl, que Ricard impugna. Eso sería innecesario si el libro de Ludwig Pfandl fuese más conocido y asequible, pero no lo es porque, hasta ahora, sólo existe la edición original en alemán; ${ }^{16}$ afortunadamente este Instituto prepara ya la edición de la traducción al español, que esperamos saldrá de prensas en el curso de 1963.

Es imposible resumir en pocas líneas lo que Pfandl explica, respecto al Sueño, en casi cuarenta cuartillas. Desde luego, reconoce y admira la arquitectura del poema, del que dice que "es un tríptico gótico". En su estructura distingue cinco partes: el sueño mágico (es el sueño, o sea el dormir, del cosmos) ; la teoría del sueño ( es el sueño, dormir, fisiológico o del cuerpo); la intuición del sueño (la ensoñación del alma que mira las cosas y quiere entender la creación); el paso al umbral del sueño (el despertarse del sér humano); el nacimiento del sol (el despertar del cosmos).

No parece, por lo que él mismo dice, que Pfandl haya supuesto que Sor Juana quiso contarnos un sueño real; en todo caso Pfandl supone que ese sueño, en cuanto tal, fue solamente lo que en el poema es su parte central, la que él llama "la intuición del sueño"; él dice que todo lo demás "es visión imaginada y poesía, sino que también es en su mayor parte poesía del inconsciente. Ni la magia nocturna, ni la teoría del sueño, ni el traspaso del umbral del sueño, ni tampoco el nacimiento del sol fueron realmente soñados por Juana Inés, sino que fueron posteriormente poetizados por ella, fantaseando y soñando despierta, para la pieza esencial, para la propia experiencia del sueño, para el anhelado viaje al universo soñado por el alma. Todo el Primer Sueño es un ardiente y centelleante haz de rayos divergentes de deseos que saltan del núcleo ígneo de un único anhelo de rafz primordial: el deseo infantil reprimido y el desenfrenado afán de saber..."17

15 Rémy de Gourmont: "La création subconsciente", dans La Culture des Idées 20ème edition. Mercure de France, Paris, 1946. pp. 45-46.

16 Ludwig Pfandl: Die Zehnte Muse von Mexico Juana Inés de la Cruz. Ihr Leben, Ihre Psyche. Muenchen, 1946.

17 Pfandl: $O p$ cit., traducción inédita. 
En cuanto a considerar el poema como un testimonio psicológico (uno de los puntos de su estudio que Ricard reprocha a Pfandl), este autor sin duda lo hace, con gran detalle y como tal lo aprecia mucho: "en el círculo de la literatura universal -dice-, no se dan muchas obras poéticas en las cuales hayan dejado fluir sus creadores, como Juana Inés lo ha hecho aqui, tan integramente toda su vida interior y todo su dolor espiritual." 18 Repito que no sería posible resumir el largo y profundo análisis que hace del poema, el cual, por lo demás, tampoco podría desglosarse del resto del libro, pues mucho padecería su posible interpretación, ya que el estudio de Pfandl es un todo con gran unidad y hay que conocerlo así. Por otra parte el objeto de estas páginas es comentar el estudio del Dr. Ricard; por todo ello creo que basta con lo tan esquemáticamente esbozado, simplemente para completar el sentido de algunas frases de Ricard antes citadas.

Ricard completa su explicación o interpretación y crítica del poema refiriéndolo, como dije atrás, a otros ejemplos literarios; esas tres páginas finales de su segunda lección son altamente instructivas por las varias direcciones a que apuntan; creo que los estudiosos de estos temas reconocerán su valor, por lo que hago de ellas otra más de estas transcripciones del texto francés:

"Il est probable que la critique aurait plus facilement résisté aux dangereuses tentations que nous avons déplorées si l'on avait moins oublié que le Sueño, dans son inspiration générale, se rattache à un genre ancien et parfaitement défini. Je ne pense pas à ces innombrables songes qui pullulent dans nos littératures, qui ne sont le plus souvent qu'un procédé d'exposition ou un procédé dramatique, et que l'on peut appeler les songes littéraires. Pour me borner à la littérature espagnole, le Sueño de Sor Juana n'a pas grand' chose de commun avec le rêve de Don Quichotte dans la cueva de Montesinos ni avec les Sueños de Quevedo, ni avec le thème de La vida es sueño de Calderón, ni même avec le Somnium où, au $\mathrm{XVe}$ siècle, Juan Maldonado nous décrit l'Amerique chrétienne et idyllique qu'il a vu en songe. On n'y trouve même qu'un écho bien affaibli d'une silva connue de Quevedo, El sueño, où il est d'ailleurs question du sommeil et non du rêve. Je pense à autre chose. Je pense à une autre tradition, où le rêve est décrit en lui-

18 Pfandl: Op cit., traducción inédita. 
même, pour sa valeur didactique, d'une manière qui n'est pas sans rapport avec le range médiéval de la visión. C'est la tradition du songe philosophique. Ce songe relate souvent une ascension, d'où son nom de songe d'anabasis, et le Corpus hermeticum occupe une place importante dans son histoire et dans son dévelopement. Sor Juana n'a pas pu ne pas connaître cette tradition, et elle a certainement connu son représentant le plus illustre, le Songe de Scipion de Gicéron (rappelons-nous qu'il y en a une reminiscence dans la première Elégie de Garcilaso). Scipion, le principal interlocuteur du De Republica (dont le Songe de Scipion n'est qu'un fragment) raconte un songe durant lequel il a été transporté parmi les étoiles. Il dépeint Ia splendeur nocturne du ciel et le spectacle de la terre qu'il aperçoit toute petite à ses pieds. Son grand-père Scipion l'Africain l'accueille et lui dévoile les mystères de l'univers et la destinée des âmes après la mort. A côté d'un aspect cosmique, il y a donc dans cet écrit un aspect eschatologique -sort futur des justes et des méchants- et un aspect moral - petitesse de la terre et vanité des choses humaines-. Il ne nous intéressent pas en ce moment, car ils sont absents du Sueño: Sor Juana -et ce trait est bien d'elle- est restée sur le plan intellectuel, sur le plan de la connaissance. Les affinités entre l'oeuvre de Cicéron et la sienne ne dépassent pas ce domaine. Mais elles existent. Or il se trouve que le Songe de Scipion n'est lui-même qu'un écho de toute une littérature et qu'il aura à son tour d'innombrables échos et prolongements dans la littérature grecque et latine, à tel point que dans son Icaroménippe Lucien éprouvera le besoin de caricaturer ces voyages célestes.

"Assurément, sauf le texte de Cicéron et peut-être celui de Lucien, Sor Juana n'a dû connaître cette littérature que de seconde main; encore faut-il noter que celle-ci est fortement teintée de platonisme et de néo-platonisme et que la religieuse mexicaine avait bien des moyens de s'initier à ce vaste courant, ne serait-ce qu'à travers les Diálogos de Amor de Léon Hébreu, traduits d'italien en castillan par l'Inca Garcilaso en 1586. Mais cela ne l'a pas empêchée de rester elle-même. Nous remarquions tout à l'heure qu'elle a laissé de côté les spéculations eschatologiques et les lieux communs moraux, les toppi, qui faisaient partie du genre dans l'Antiquité. Elle a laissé pareillement de côté un autre élément qui apparaît presque toujours dans la littérature gréco-latine: le 
guide, l'initiateur, qui dirige le songeur et lui révèle les mystéres du monde et de la vie: Scipion l'Africain dans le Songe de Scipion, le dieu Noûs dans le Pimandre du Corpus hermeticum. ${ }^{19}$ Chez Sor Juana, l'esprit humain demeure seul, abandonné à ses propres forces -symbole de sa propre formation solitaire-, et il s'y joint toutes sortes d'éléments qui proviennent soit de ses lectures, soit de son expérience personnelle. Si j'ai insisté sur cette littérature du songe philosophique, c'est que jamais, à ma connaissance, le Sueño n'a été replacé dans cette tradition. Mais on aurait tort d'exagérer la portée du rapprochement et de mécconnaître l'originalité foncière du poéme.

"Il suffit d'ailleurs de faire quelques constatations simples. Qu'on reprenne le Songe de Scipion: outre que, par son étendue, le texte ne représente guère que la moitié de celui de Sor Juana, il est très différent par bien des côtés; la contemplation du monde y tient una place beaucoup moins grande. Nous avons noté que les spéculations eschatologiques demeurent étrangères à Sor Juana: sa foi lui fournissait une réponse suffisante. Enfin, chez les Grecs et les Latins, il ne s'agit pas de poésie: il n'y a pas de commune mesure entre la prose élégante de Platon et de Cicéron, la mauvaise prose du Corpus hermeticum et les vers gongorinos de Sor Juana. Il faut laisser aux choses leurs véritables proportions: on ne doit parler que d'une inspiration générale qui n'exclut aucunement la création personnelle. ${ }^{20}$

Termina Ricard esa su $2^{n}$ lección reconociendo que una obra tan singular como lo es el Sueño, inevitablemente dará origen a las más divergentes interpretaciones. En cuanto a otras fuentes o influencias: "C'est aux historiens des sciences et de la philosophie - je pense aux recherches de M. Gilson sur les éléments médiévaux chez Rabelais et chez Descartes-- qu'il appartiendrait surtout de démêler cet écheveau embrouillé que constitue le système cosmologique et physio-psychologique qui guide Sor Juana dans ses visions. Eux seuls pourraient avec sûreté déceler ce que notre poétesse doit à l'alexandrinisme et aux différentes formes du platonisme, à la scolastique de source aristotélicienne, et peut-être à des

19 Sobre esto, el autor da las siguientes referencias, en notas: $A$. J. Festugière: Corpus Hermeticum. Coll. Budé, Paris, 1954 y Festugière: La révélation d'Hermès Trismégiste. Le Dieu cosmique, Paris, 1949.

20 Ricard: Une poétesse... pp. 24-26. 
philosophies plus récentes qui commençaient à peine à pénétrer en Nouvelle-Espagne ..." 21

La $3^{a}$ lección se titula: La poésie religieuse de Sor Juana et la société mexicaine au xvire siècle. En esa poesía distingue la de manera "culta" y la popular, pero asi como el Sueño es ejemplo típico de poesía culta, profana, de igual modo el ejemplo típico de la manera popular son los villancicos. Da, luego, breves y precisos antecedentes del villancico, para que su auditorio ubique el género y señala dos tendencias en su evolución: "D'une part, on a vu surgir des parties dialoguées qui constituent comme un embryon de drame lyrique (on a parlé d'opereta sacra). D'autre part, ces poésies, profanes à l'origine, on été de plus en plus transposées a lo divino." 22

Naturalmente también alude a la forma métrica, a las circunstancias en que se usaban los villancicos (primero en la noche de Navidad y luego en muy diversas festividades religiosas). Después trata especialmente de los villancicos que compuso Sor Juana; señala que la poetisa, con frecuencia, acentuaba deliberadamente el carácter teatral al que tendía aquel género, ya sea por medio del reiterado empleo de diálogos ficticios o bien, en mayor número de ocasiones, por la intervención de personajes diferentes cada uno de los cuales canta, a su turno, su pequeña parte.

Se refiere al uso de diversos idiomas, tan característico en esos poemitas de Sor Juana, mezcla de lenguas que suele ser un elemento burlesco; procedimiento, dice Ricard: "qui évoque à la fois Gil Vicente et Molière et qui fait aussi pensar au gracioso de la comedia..." 23

Otras muchas observaciones críticas y eruditas referencias hay en esta parte última, que ojalá no fuera tal, pues cuánto deseariamos que el doctor Robert Ricard prosiguiera dándonos sus perspicaces y luminosos comentarios sobre la obra y la personalidad de Sor Juana Inés de la Cruz, tema que conoce tan fina y profundamente como lo demuestran las páginas de ese curso dictado en la Sorbona, del que aquí apenas he podido hacer un esbozo y entresacar algunos fragmentos de su precioso contenido.

21 op. cit., p. 27.

22 op. cit., p. 32.

23 op. cit., p. 37. 